\title{
Prevention of ultraviolet radiation-induced immunosuppression by sunscreen in Candida albicans-induced delayed-type hypersensitivity
}

\author{
QUAN CHEN ${ }^{1}$, RUNXIANG LI $^{1}$, XIAOXIA ZHAO ${ }^{2}$, BIHUA LIANG $^{1}$, \\ SHAOYIN MA ${ }^{1}$, ZHENJIE LI $^{1}$ and HUILAN ZHU ${ }^{1}$ \\ ${ }^{1}$ Guangzhou Institute of Dermatology, Guangzhou, Guangdong 510095; ${ }^{2}$ Department of Dermatology, \\ Haikou City People's Hospital, Haikou, Hainan 570208, P.R. China
}

Received April 7, 2015; Accepted March 10, 2016

DOI: $10.3892 / \mathrm{mmr} .2016 .5284$

\begin{abstract}
Ultraviolet (UV) radiation-induced immunosuppression leading to skin cancer has received increased attention in previous years. The present study aimed to investigate the immunoprotection offered by Anthelios sunscreen in a mouse model of Candida albicans-induced delayed-type hypersensitivity. Anthelios sunscreen was applied to the skin on the dorsal skin of BALB/c mice treated with a sub-erythema dose of solar-simulated radiation. Delayed-type hypersensitivity was induced by immunization with Candida albicans. Changes in the skin thickness of the foot pads were measured, and immunosuppression rates were also evaluated. The expression levels of CD207, CD80 and CD86 in the Langerhans cells were semi-quantitatively detected using Western blotting and immunohistochemical assays. The delayed-type hypersensitivity mouse model was successfully established. The minimal erythema doses of UVA and UVB exposure to the mice were 2,000 and $145 \mathrm{~mJ} / \mathrm{cm}^{2}$, respectively. The immunosuppression rates in the sunscreen group and non-sunscreen group were 24.39 and $65.85 \%$, respectively $(\mathrm{P}<0.01)$. The results of the Western blotting and immunohistochemistry showed that the expression levels of CD207 $(\mathrm{P}<0.01), \mathrm{CD} 80(\mathrm{P}<0.05)$ and $\mathrm{CD} 86(\mathrm{P}<0.01)$ were higher in the sunscreen group, compared with those in the non-sunscreen group. UV exposure reduced Candida albicans antigen-induced delayed-type hypersensitivity. Anthelios sunscreen was found to protect the skin from immunosuppression through the activation of epidermal Langerhans cells.
\end{abstract}

Correspondence to: Dr Huilan Zhu, Guangzhou Institute of Dermatology, 56 Hengfu Road, Guangzhou, Guangdong 510095, P.R. China

E-mail: zhlhuilan@126.com

Key words: immunosuppression, ultraviolet, sunscreen, Langerhans cells

\section{Introduction}

Solar ultraviolet radiation (UVR) has been demonstrated to have systemic suppressive effects on the immune system. UVR can suppress immune reactions at a local and systemic level $(1,2)$. One of the major molecular mediators of photoimmunosuppression is UVR-induced DNA damage (3). It has also been shown that chronic UVR can lead to the induction of skin cancer, which is the most serious adverse health effect of UVR (4). Excess sun exposure increases the risk of non-melanoma and melanoma skin cancer $(5,6)$. It has been estimated by the United Nations Environment Panel that, in the last few years, $>2,000,000$ cases of non-melanoma and 200,000 cases of malignant melanoma have occurred annually worldwide (7). UVR inhibits antigen presentation and induces the release of immunosuppressive cytokines, and this specific immunosuppression is mediated by antigen-specific suppressor/regulatory T cells. Langerhans cells are considered to be the exclusive antigen-presenting cells in the skin, and Langerhans cells have been found to be depleted upon UVR exposure $(8,9)$. However, the precise mechanism underlying the induced immunosuppression remains to be elucidated (10).

The use of sunscreen has been shown to have a protective effect against sun exposure, and several studies have indicated that sunscreens afford a level of protection against UVR-induced immunosuppression $(11,12)$. Thus, in addition to sunburn protection factor (SPF), the determination of the immune protection factor (IPF) has been suggested as an alternative measurement $(13,14)$, which may reflect immune protection effect more accurately. The majority of previous mouse models evaluating the immunoprotective effects of sunscreen have been directly based on SPF parameters, indicating the contact hypersensitivity $(15,16)$. The present study aimed to investigate the immunoprotection offered by Anthelios sunscreen in a Candida albicans-induced delayed-type hypersensitivity mouse model, which demonstrates a protective localized cell-mediated immune response and has been widely used in investigations to assess immune responses $(17,18)$. The present study aimed to clarify the effect of Anthelios sunscreen on UVR-induced immunosuppression and provide evidence to support the application of sunscreen to prevent UVR-induced skin injury. The 
present study provided evidence to support the application of sunscreen in the prevention UVR-induced skin injury.

\section{Materials and methods}

Animals. The current study was approved by approved by the animal ethics committee of Sun Yat-sen University (Guangzhou, China). Pathogen-free BALB/c mice (6-week-old; body weight, $15 \pm 1 \mathrm{~g}$ ) were purchased from the Animal Center of Sun Yat-sen University (Guangzhou, China). The animals were housed in a specific pathogen-free grade, forced air laboratory under standard natural lighting conditions ( $12 \mathrm{~h} \mathrm{light} / 12 \mathrm{~h}$ dark) at $20-24^{\circ} \mathrm{C}$, and were provided with food and water. During the entire experimental process, all efforts were made to minimize the suffering of the animals, in accordance with the National Institute of Health Guidelines for the Care and Use of Laboratory Animals (19).

Reagents and equipment. The UVA, UVB and solar-simulated (ss)UVR spectra were produced using an SUV-1000 Solar UV simulator (Sigma-Aldrich, St. Louis, MO, USA). The intensity $\left(\mathrm{mJ} / \mathrm{cm}^{2}\right)$ of the UV output was measured using a PMA2100 radiometer (Solar Light Company, Glenside, PA, USA), and the timing of the UV exposure was adjusted using a timing device. The spectral output of the solar simulator was measured using an OL-754 spectroradiometer (Optronic Laboratories, Orlando, FL, USA). Formalin-fixed Candida albicans was prepared by the Biochemistry Laboratory of Sun Yat-sen University. Sunscreen Anthelios XL (SPF 50; PPD 28) was provided by L'Oreal (Paris, France). Antibodies were purchased from commercial suppliers, as indicated below.

Dose-response to UVR. The present study first investigated the minimal erythema dose (MED) of UVA and UVB exposure to the mice by exposing the animals to various doses of ssUVR. A total of 120 mice were randomly divided into a sunscreen group and a non-sunscreen group. These groups were each divided into six subgroups $(n=10)$, which were treated with different doses of UVR. An area measuring $\sim 8 \mathrm{~cm}^{2}$ of the dorsal skin of the mice was shaved using hair clippers 1 day prior to UV irradiation, and the skin was covered with or without sunscreen $\left(2 \mathrm{mg} / \mathrm{cm}^{2}\right)$. The other body areas were protected from UV exposure. The dorsal skin was then exposed to the sunlight system with various doses of ssUVR (UVA, 1,000-3,500 $\mathrm{mJ} / \mathrm{cm}^{2}$; UVB: $30-1,200 \mathrm{~mJ} / \mathrm{cm}^{2}$ ) for $60 \mathrm{sec}$. The pre-UV and post-UV skin thickness was measured using hand-held Episcan-1-200 high frequency ultrasound (Longport International, Silchester, UK). The changes in skin thickness were plotted to obtain the response curves against various UVR doses under a constant sunscreen dose. The minimum dose of ssUVR required to cause a significant increase in skin thickness in the sunscreen group, compared with the non-sunscreen group, was used for further experiments, with a factor of 0.7 .

Immunization with Candida albicans. The experimental design is shown in Fig. 1. A total of 40 mice were randomly divided into four groups $(n=10)$ : Group A (Sunscreen+UV+immunization), Group B (UV+immunization), Group C (positive control) and Group D (negative control). The mice were anesthetized with $10 \%$ chloral hydrate (Sinopharm Chemical Reagent Co., Ltd,
Shanghai, China) by intraperitoneal injection at a dose of $280-350 \mathrm{mg} / \mathrm{kg}$. For the UV groups, 0.7X MED was provided to individual animals, and this procedure was repeated for 5 days. On the fifth day, the mice were immunized by subcutaneous injection of $10^{7}$ formalin-fixed Candida albicans to each foot pad as previously reported (20). After $24 \mathrm{~h}$, the thickness of each foot was measured with vernier calipers and the mean footpad swelling in each mouse was calculated as follows: Mean swelling $=($ left foot swelling + right foot swelling $) / 2$, according to a previous study (20). Additional mice, which received UV treatment only and immunization only were used as a negative control and positive control, respectively. The increase in skin thickness, compared with the negative control was used to normalize data. Immunosuppression rates were calculated as: Immunosuppression rate $=(1-$ experimental accurate edema value / positive control of accurate edema value) $\mathrm{x} 100 \%$.

Determining expression levels of CD207, CD80 and CD86 using western blot analysis. Following measurements of the thickness of each foot, the present study investigated the expression levels of CD207, CD80 and CD86, which indicate immune responses from Langerhans cells. Biopsy specimens ( $2 \mathrm{~g}$ ) from the UV-exposed dorsal skin were dissected to extract total protein and the mice were then sacrificed by decapitation. The skin tissue from each group was homogenized in radioimmunoprecipitation assay buffer with protease inhibitor cocktail (Santa Cruz Biotechnology, Inc., Santa Cruz, CA, USA). Total tissue extracts were separated by centrifugation at $12,000 \mathrm{x}$ g and $4^{\circ} \mathrm{C}$ for $30 \mathrm{~min}$. Subsequently, $5 \mu \mathrm{g}$ of the total tissue extract was resolved by $12 \%$ sodium dodecyl sulfate polyacrylamide gel electrophoresis and electrotransferred onto a polyvinylidene difluoride membrane (Whatman; GE Healthcare Life Sciences, Chalfont, $\mathrm{UK})$ at $100 \mathrm{~V}$ for $1 \mathrm{~h}$. The membranes were blocked in 5\% non-fat milk (Amresco, LLC, Solon, OH, USA) with shaking at room temperature for $1 \mathrm{~h}$, then incubated with monoclonal rat anti-mouse CD207/Langerin (1:2,000; cat. no. 14-2073; eBioscience, Inc., San Diego, CA, USA), polyclonal rabbit anti-mouse CD80 (1:2,000; cat. no. bs-2211R; BIOSS, Beijing, China), polyclonal rabbit anti-mouse CD86 (1:2,000; cat. no. bs-1035R; BIOSS) and monoclonal rabbit anti-actin (1:2,000; cat. no. 04-1040; EMD Millipore, Billerica, MA, USA) antibodies at room temperature for $2 \mathrm{~h}$. Following 3 washes in $1 \mathrm{X}$ Tris-buffered saline with $1 \%$ Tween 20 (TBS-T; Guangzhou Whiga Technology Co., Ltd., Guangzhou, China) for $10 \mathrm{~min}$, the PVDF membranes were then incubated with polyclonal anti-rat horseradish peroxidase (HRP)-conjugated IgG (1:10,000; cat. no. AP136P; EMD Millipore) and anti-rabbit HRP-conjugated IgG (1:10,000; cat. no. 12-348; EMD Millipore) at room temperature for $30 \mathrm{~min}$. Antibodies were diluted in TBS-T. The Western blotting experiments were performed using biological replicates. The immunoblot bands were visualized using an enhanced chemiluminescence method (Thermo Fisher Scientific, Waltham, MA, USA) and quantified using Image J software (version 1.49; National Institutes of Health, Bethesda, MD, USA).

Immunohistochemistry. The biopsy specimens obtained from the UV-exposed back skin were dissected immediately 


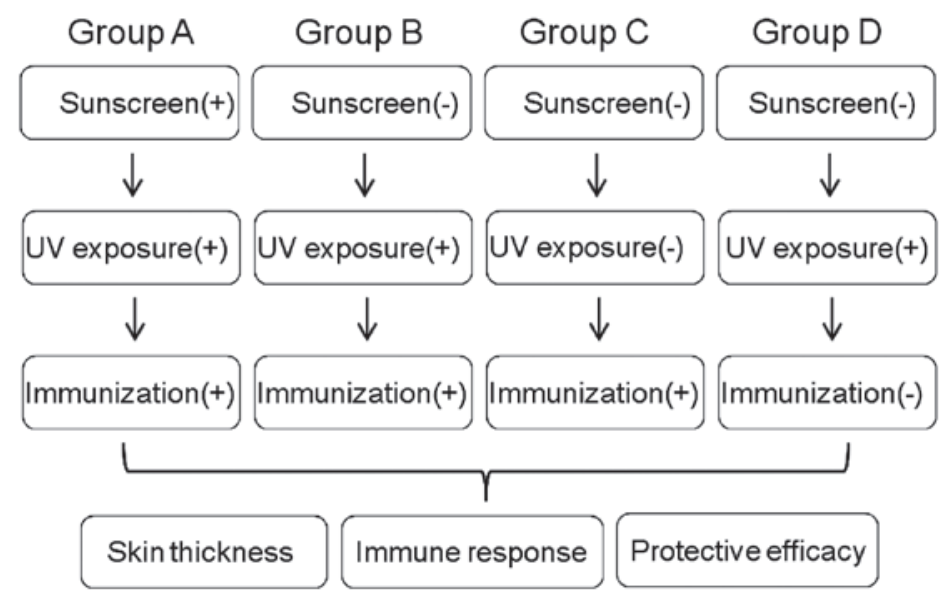

Figure 1. Schematic diagram showing the experimental design of the present study. Mice were randomly divided into four groups ( $n=10)$ : Group A (sunscreen+UV+immunization), Group B (UV+immunization), Group C (positive control) and Group D (negative control). Mice were anesthetized and prepared for UV radiation. For the UV groups, $0.7 \mathrm{X}$ minimal erythema dose was repeatedly administered to the individual animals for 5 days. On the fifth day, the mice were immunized by subcutaneous injection of $10^{7}$ formalin-fixed Candida albicans to each foot pad. Additional mice were treated with UV treatment only or immunization only as a negative control and positive control, respectively. At 24 h-post challenge, the thickness of each foot was measured using vernier calipers and the mean footpad swelling for each mouse was calculated. The increase in skin thickness from that in the negative control was used to normalize data. UV, ultraviolet.

A

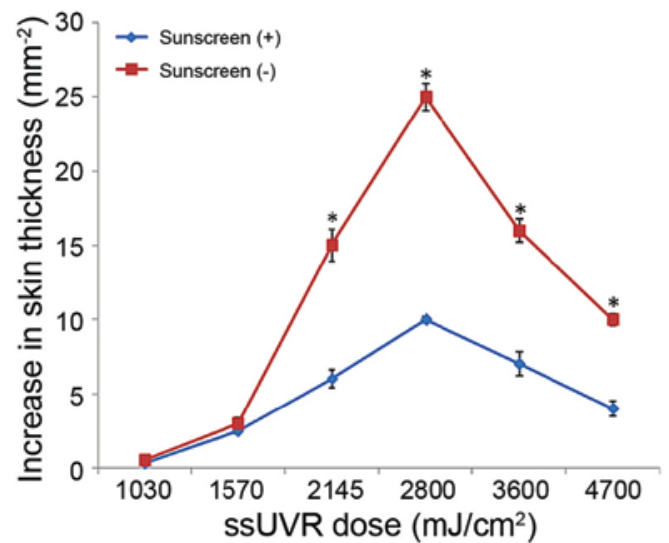

B

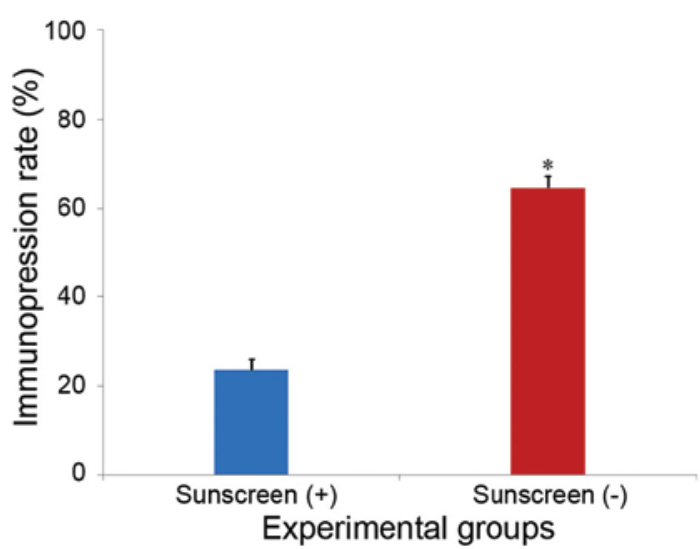

Figure 2. Dose-response curve showing the increase in skin thickness against UVR. Mice were randomly divided into a sunscreen group and a non-sunscreen group. Each group was divided into six subgroups $(n=10)$, each treated with a different dose of UVR. Prior to UVR, an area of $\sim 8 \mathrm{~cm}{ }^{2}$ of the dorsal skin of the mice was covered with, or without, sunscreen $\left(2 \mathrm{mg} / \mathrm{cm}^{2}\right)$. The dorsal skin was then exposed to a sunlight system at various ssUVR doses (UVA, $1,000-3,500 \mathrm{~mJ} / \mathrm{cm}^{2}$; UVB, 30-1,200 $\mathrm{mJ} / \mathrm{cm}^{2}$ ) for $60 \mathrm{sec}$. (A) The increases in skin thickness were plotted to obtain the response curves against various ssUVR doses under a constant sunscreen dose. The minimum dose of ssUVR required to cause a significant increase ("P<0.05) in skin thickness in the sunscreen group, compared with the non-sunscreen group, was used for further experiments, (factor of 0.7). (B) The immunosuppresion rate of the sunscreen and non-sunscreen groups were 24.39 and $65.85 \%$, respectively. ${ }^{*} \mathrm{P}<0.05$ vs. sunscreen $(+)$. The data are presented as the mean \pm standard deviation. $\mathrm{UVR}$, ultraviolet radiation. ssUVR, solar-stimulated UVR.

following the measurement of footpad thicknesses. The tissues were fixed in $4 \%$ formaldehyde (Guangzhou Chemical Reagent Factory, Guangzhou, China) solution in phosphate-buffered saline (PBS) and cut using a Leica RM2235 microtome (Leica Microsystems GmbH- Wetzlar, Germany) into sections of $4 \mu \mathrm{m}$. The sections were then deparaffinized in xylene (Guangzhou Chemical Reagent Factory), and hydrated in a series of ethanol and distilled water. Endogenous peroxidase was eliminated by incubating the sectioned tissues in $5 \% \mathrm{H}_{2} \mathrm{O}_{2}$ in methanol for $30 \mathrm{~min}$. Non-specific staining was blocked by incubation in 5\% normal mouse serum in PBS for $30 \mathrm{~min}$ at $37^{\circ} \mathrm{C}$. The sections were then incubated overnight at $4^{\circ} \mathrm{C}$ with primary antibodies, including mouse anti-CD207/Langerin, anti-CD80 and anti-CD86 antibodies (1:200). Following rinsing three times for 5 min with PBS, the sections were incubated with HRP-conjugated anti-mouse $\operatorname{IgG}(1: 2,000)$ for $1 \mathrm{~h}$. The sections were rinsed with PBS twice for $10 \mathrm{~min}$, following which the slides were developed using 3,3'-diaminobenzidine (Beijing Biosynthesis Biotechnology). The sections were examined using a Nikon Eclipse TE2000-U light microscope (Nikon Corporation, Tokyo, Japan), and images captured with a digital camera.

Statistical analysis. SPSS version 13.0 software (SPSS, Inc., Chicago, IL, USA) was used in the present study for all statistical analyses. The results are presented as the mean \pm standard deviation. The increases in skin thickness in the groups were 


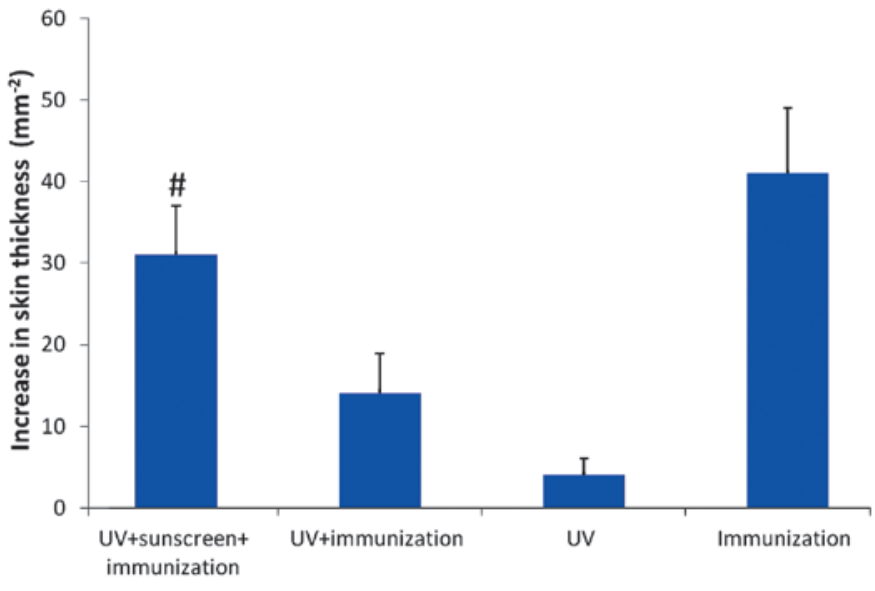

Experimental groups

Figure 3. Comparison of the increase in skin thickness between different groups. Mice in treated with or without sunscreen were injected with formalin-fixed Candida albicans. At $24 \mathrm{~h}$-post challenge, the thickness of each foot was measured using vernier calipers and the mean footpad swelling of each mouse was calculated as follows: Mean swelling $=($ left foot swelling + right foot swelling) $/ 2$. The increase in skin thickness from that of the negative control was used to normalize data. Data are presented as the mean \pm standard deviation. ${ }^{\#} \mathrm{P}<0.01$, compared with the non-sunscreen group. UV, ultraviolet.

A

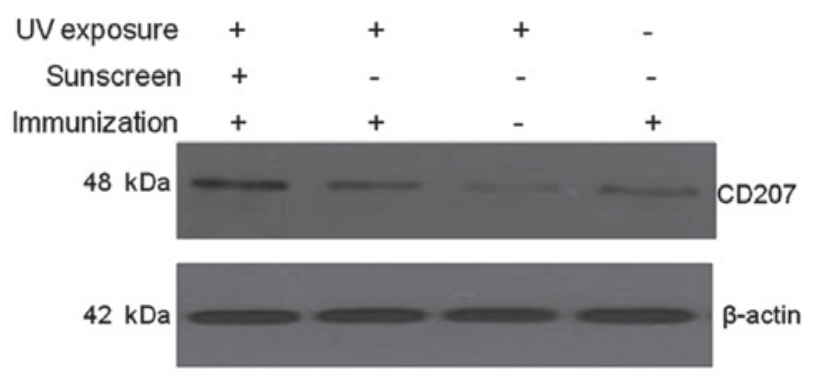

B

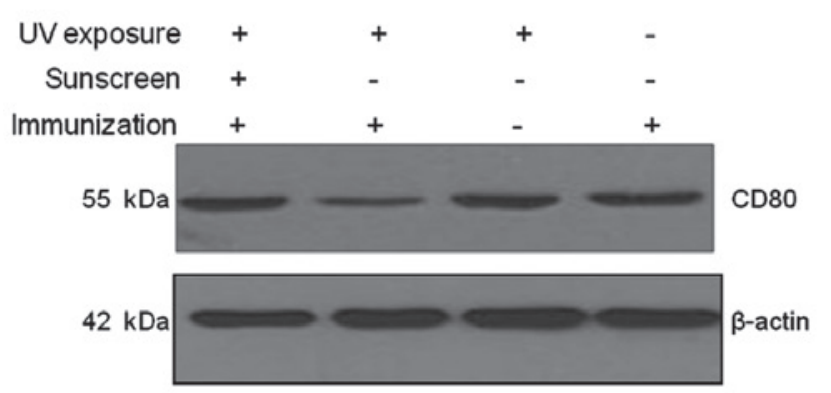

C

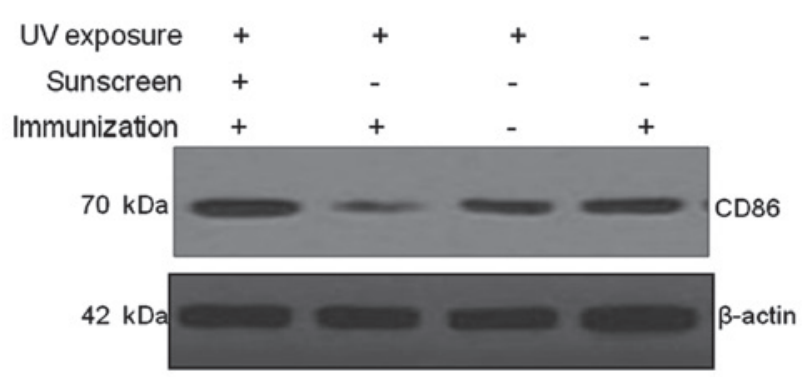

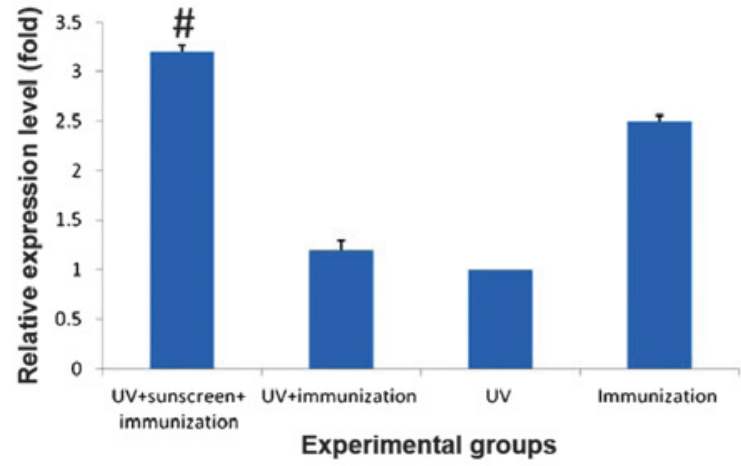
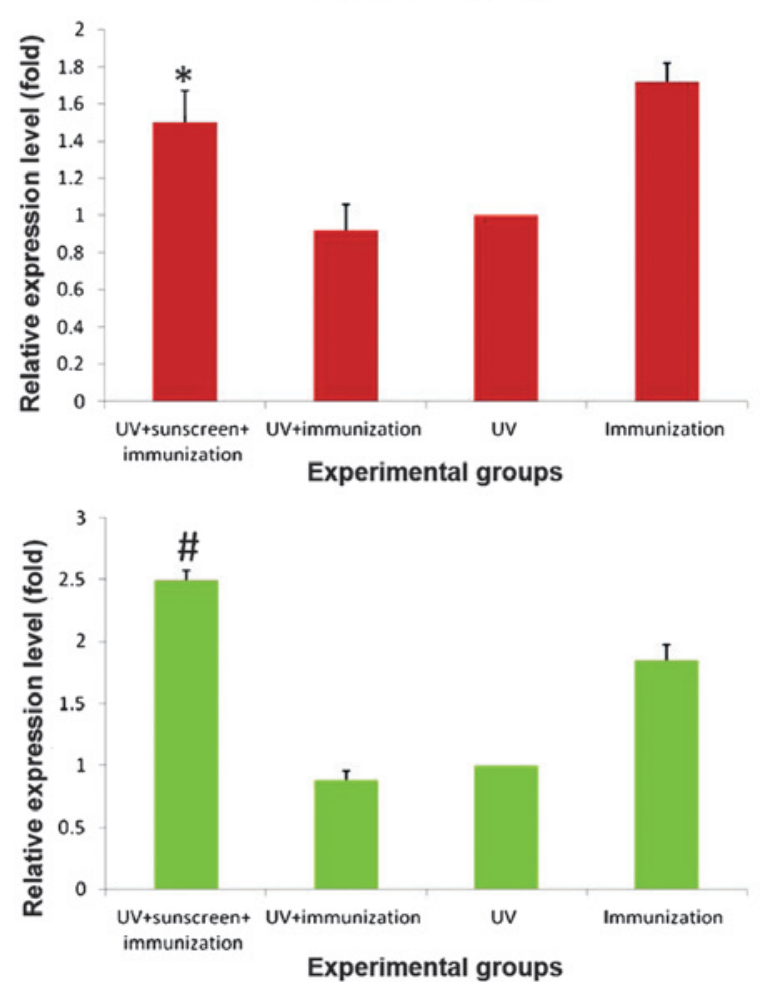

Figure 4. Western blot analyses of the expression levels of CD207, CD80 and CD86. Compared with the positive control, the expression level of (A) CD207, (B) CD80 and (C) CD86 in the non-sunscreen group was significantly decreased, whereas the use of sunscreen upregulated the expression of CD207, CD80 and $\mathrm{CD} 86(\mathrm{P}<0.05)$.Data are presented as the mean \pm standard deviation. ${ }^{*} \mathrm{P}<0.05$ and ${ }^{*} \mathrm{P}<0.01$, compared with the non-sunscreen group. UV, ultraviolet. 


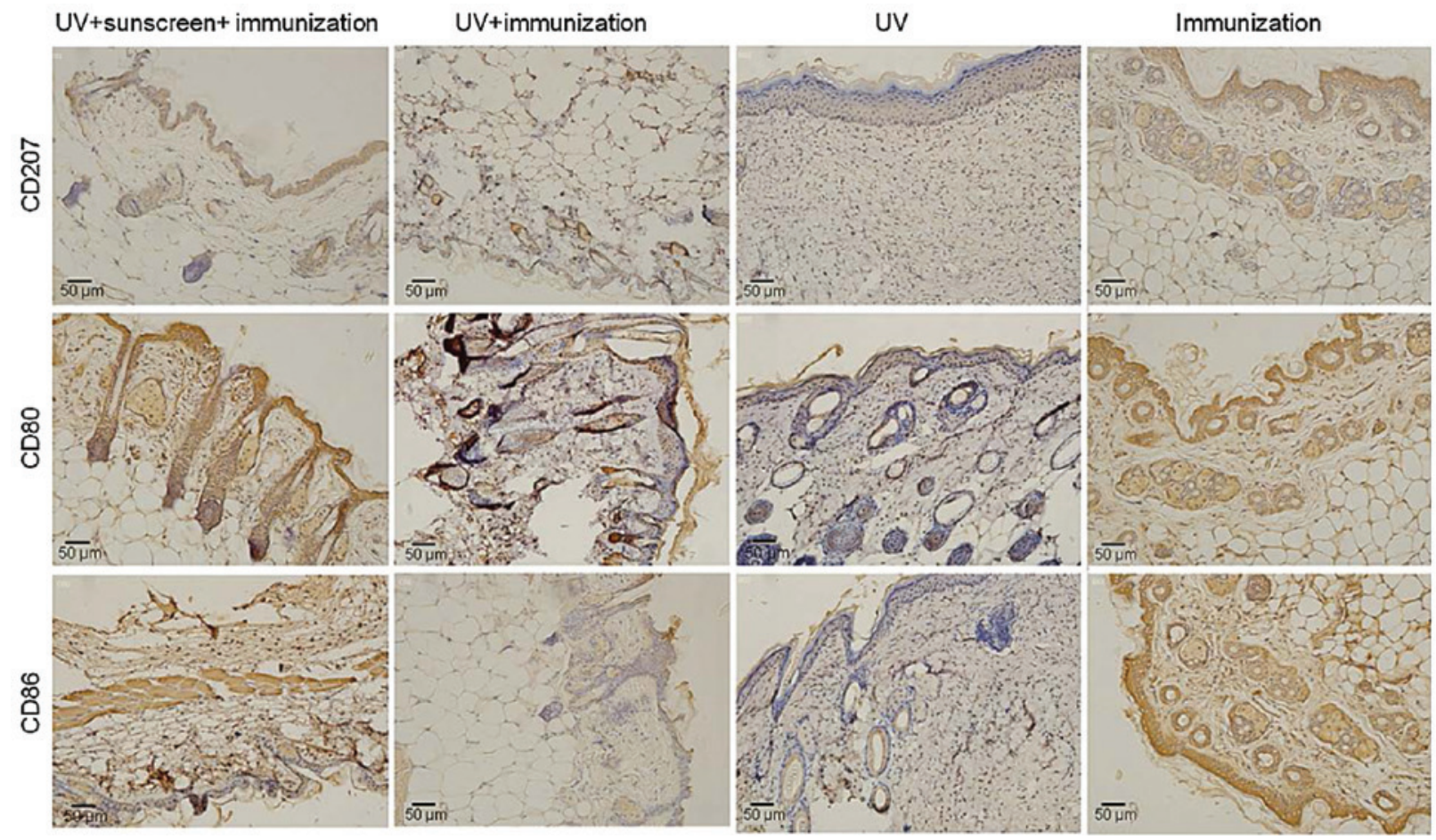

Figure 5. Immunohistochemical analysis demonstrating the expression levels of CD207, CD80 and CD86. CD207, CD80 and CD86 were highly expressed in the positive control (immunization group), and markedly suppressed by UVR treatment, while the expression levels of these immune molecules were recovered by the sunscreen treatment. Sections were examined by light microscopy (magnification, x100; scale bar=50 $\mu \mathrm{m}$ ). UVR, ultraviolet radiation.

compared using Student's t-test. $\mathrm{P}<0.05$ was considered to indicate a statistically significant difference.

\section{Results}

Dose-response to UVR. To determine the MED value of the UVR, preliminary experiments were performed, in which animals were exposed to various doses of ssUVR. Response curves were drawn by plotting the increases in skin thickness against various ssUVR doses under a constant sunscreen dose. The dose response curves from the sunscreen group and non-sunscreen group are shown in Fig. 2. The dose of ssUVR varied between 1,030 and $4700 \mathrm{~mJ} / \mathrm{cm}^{2}$. The increase in skin thickness started to differentiate from $1,570 \mathrm{~mJ} / \mathrm{cm}^{2}$, and a significant difference was found at $2,145 \mathrm{~mJ} / \mathrm{cm}^{2}(\mathrm{P}<0.05)$, at which UVA and UVB were 2,000 and $145 \mathrm{~mJ} / \mathrm{cm}^{2}$, respectively. In consideration of animal ethics, a 0.7X MED was set as the ssUVR dose, of which UVA and UVB were 1,400 and $101.5 \mathrm{~mJ} / \mathrm{cm}^{2}$, respectively.

Immunosuppression. Overall, the increases in skin thickness of Group A (sunscreen+UV+immunization) and Group C (positive control) were higher, compared with Group B (UV+immunization) and Group D (negative control), indicating UVR-induced immunosuppression in the Candida albicans-induced delayed-type hypersensitivity mouse model was successfully established (Fig. 3). The severity of foot pad swelling in the sunscreen group was significantly higher, compared with that in the non-sunscreen group $(\mathrm{P}<0.01)$. The immunosuppresion rate of the sunscreen group and non-sunscreen group were 24.39 and $65.85 \%$, respectively.
Western blot analysis. As shown in Fig. 4, the expression level of CD207 was measured in biopsy specimens from UV-exposed dorsal skin. Compared with the positive control, the expression level of CD207 in the non-sunscreen group was significantly decreased, whereas the use of sunscreen upregulated the expression of CD207 (P<0.01; Fig. 4A and B). Accordingly, the present study further measured the expression levels of CD80 and CD86, which also represent immune responses of Langerhans cells. The results of the Western blotting showed that, compared with the non-sunscreen group, the expression levels of CD80 ( $\mathrm{P}<0.05$; Fig. $4 \mathrm{C}$ and $\mathrm{D})$ and CD86 $(\mathrm{P}<0.01$; Fig. $4 \mathrm{E}$ and $\mathrm{F}$ ) were recovered by the sunscreen treatment.

Immunohistochemistry results. To confirm the effect of sunscreen on the immune responses against Candida albicans-induced delayed-type hypersensitivity, the present study performed an immunohistochemical assay to measure the expression levels of CD207, CD80 and CD86 in the treatment groups. The results demonstrated that these molecules were expressed at high levels in the positive control (immunization group; Fig. 5), whereas the expression levels were markedly suppressed by UVR treatment. Compared with the non-sunscreen group (UV+immunization group), the expression levels of these immune molecules were maintained in the sunscreen group (UV+sunscreen+immunization; Fig. 5), which was consistent with the results of the western blot analysis.

\section{Discussion}

The association between over-exposure to UVR and increasing incidence of skin cancer has been investigated in previous years. Photoimmunosupression of adaptive immune 
responses is important, however, the pathways involved are complex and remain to be fully elucidated (21). Sunscreen is an effective and available agent for protection against light damage in dermatological practice. The majority of evaluation methods for sunscreen protection is an SPF-based measure of UVB-induced erythema response, however, this fails to provide an appropriate evaluation of immune protection. IPF has been suggested for years as an immune protection indicator in sunscreen (6). However, the lack of appropriate measurements to evaluate immunosuppression remains a challenge in studies in photodermatology. Kim et al examined the UVB-induced erythema response in hairless mice for 5 days following the injection of Candida albicans, and found that the Candida albicans-induced delayed-type hypersensitivity response in the mouse model is similar to the response to sunlight in humans (19). In the present study, BALB/c mice were irradiated using ssUVR, and showed that a sub-erythema dose of ssUVR exposure caused immune suppression. To determine the MED value of UVR, preliminary experiments were peroformed, in which animals to various dose of ssUVR. The ssUVR dose was set at 0.7X MED for the further experiments. It was found that the immunosuppression rate of the sunscreen group $(24.39 \%)$ was significantly lower, compared with that of the non-sunscreen group $(65.85 \%)$.

The present study investigated the potential mechanism involved by measuring the expression levels of CD207, CD80 and CD86 in biopsy specimens of the UV-exposed dorsal skin. The results showed that the expression levels of CD207, CD80 and CD86 were higher in the sunscreen group, compared with the non-sunscreen group. CD207/langerin is a type II membrane-associated C-type lectin, known to be expressed exclusively by Langerhans cells (22). When it is activated, it expresses membrane-associated antigens CD80 and CD86, which are $\mathrm{T}$ cell co-stimulatory molecules. These can be combined with the excitatory CD28 receptor and inhibitory CTLA-4 receptor $(23,24)$. It is generally considered that a large quantity of CD86 is expressed rapidly following antigen activation of Langerhans cells. CD86 and CD80 are important in the immune response in early and late stages, respectively (25). Therefore, upon antigen injection, the expression levels of CD207, CD86 and CD80 may reflect immune responses conferred by Langerhans cells. In the present study, the results of Western blot analysis and immunohistochemistry coincidently supported the hypothesis that Anthelios sunscreen can protect the skin from immunosuppression through activating epidermal Langerhans cells.

In the present study, a Candida albicans-induced delayed-type hypersensitivity mouse model was successfully established, which was indicated by the positive control. Footpad swelling was marked in the positive control group, compared with the other groups. In addition, Langerhans cell-activating molecules were detected upon injection of Candida albicans antigen $24 \mathrm{~h}$ following challenge. Considering the value of the Candida albicans-induced delayed-type hypersensitivity mouse model in the immune response, this model may be used to evaluate the protective efficacy of other commercial sunscreens. Taken together, the present study demonstrated that Anthelios sunscreen prevented UVR-induced immunosuppression through activating Langerhans cells. The results of the present study provide evidence to support the application of sunscreen in the prevention of UVR-induced skin injury.

\section{Acknowledgements}

This study was supported by grants from the Natural Science Foundation of Guangdong province (grant no. 2014A030313782) and the Science and Technology Planning Project of Guangdong Province (grant. no. 2013B021800044).

\section{References}

1. Goswami S and Haldar C: Melatonin as a possible antidote to UV radiation induced cutaneous damages and immune-suppression: An overview. J Photochem Photobiol B 153: 281-288, 2015.

2. Guo B, Naish S, Hu W and Tong S: The potential impact of climate change and ultraviolet radiation on vaccine-preventable infectious diseases and immunization service delivery system. Expert Rev Vaccines 14: 561-577, 2015.

3. Schwarz T and Schwarz A: Molecular mechanisms of ultraviolet radiation-induced immunosuppression. Eur J Cell Biol 90: $560-564,2011$

4. Fernandes TR, Santos I, Korinsfky JP, Lima e Silva BS Carvalho LO and Plapler H: Cutaneous changes in rats induced by chronic skin exposure to ultraviolet radiation and organophosphate pesticide. Acta Cir Bras 29: 7-15, 2014.

5. Granstein RD and Matsui MS: UV radiation-induced immunosuppression and skin cancer. Cutis 74 (5 Suppl): 4-9, 2004.

6. Poon TS, Barnetson RS and Halliday GM: Sunlight-induced immunosuppression in humans is initially because of UVB, then UVA, followed by interactive effects. J Invest Dermatol 125: 840-846, 2005

7. Kim Y and He YY: Ultraviolet radiation-induced non-melanoma skin cancer: Regulation of DNA damage repair and inflammation. Genes Dis 1: 188-198, 2014.

8. Schwarz A, Noordegraaf M, Maeda A, Torii K, Clausen BE and Schwarz T: Langerhans cells are required for UVR-induced immunosuppression. J Invest Dermatol 130: 1419-1427, 2010.

9. Lee $\mathrm{CH}$, Wu SB, Hong $\mathrm{CH}$, Yu HS and Wei YH: Molecular mechanisms of UV-induced apoptosis and its effects on skin residential cells: The implication in UV-based phototherapy. Int J Mol Sci 14: 6414-6435, 2013.

10. Schwarz T: Mechanisms of UV-induced immunosuppression. Keio J Med 54: 165-171, 2005.

11. Moyal DD and Fourtanier AM: Broad-spectrum sunscreens provide better protection from solar ultraviolet-simulated radiation and natural sunlight-induced immunosuppression in human beings. J Am Acad Dermatol 58 (5 Suppl 2): S149-S154, 2008.

12. Serre I, Cano JP, Picot MC, Meynadier J and Meunier L: Immunosuppression induced by acute solar-simulated ultraviolet exposure in humans: Prevention by a sunscreen with a sun protection factor of 15 and high UVA protection. J Am Acad Dermatol 37: 187-194, 1997.

13. Fourtanier A, Moyal D, Maccario J, Compan D, Wolf P Quehenberger F, Cooper K, Baron E, Halliday G, Poon T, et al: Measurement of sunscreen immune protection factors in humans: A consensus paper. J Invest Dermatol 125: 403-409, 2005.

14. Cooper KD, Baron ED, LeVee G and Stevens SR: Protection against UV-induced suppression of contact hypersensitivity responses by sunscreens in humans. Exp Dermatol 11 (Suppl 1): 20-27, 2002.

15. Roberts LK and Beasley DG: Commercial sunscreen lotions prevent ultraviolet-radiation-induced immune suppression of contact hypersensitivity. J Invest Dermatol 105: 339-344, 1995.

16. Walker SL and Young AR: Sunscreens offer the same UVB protection factors for inflammation and immunosuppression in the mouse. J Invest Dermatol 108: 133-138, 1997.

17. Heriazon A, Yager JA, Sears W and Mallard BA: Induction of delayed-type hypersensitivity and interferon-gamma to Candida albicans and anti-hen-egg white lysozyme antibody as phenotypic markers of enhanced bovine immune response. Vet Immunol Immunopathol 129: 93-100, 2009.

18. Ullrich SE, Kripke ML and Ananthaswamy HN: Mechanisms underlying UV-induced immune suppression: Implications for sunscreen design. Exp Dermatol 11 (Suppl 1): 13-16, 2002. 
19. National Research Council (US): Guide for the Care and Use of Laboratory Animals. National Academies Press, Washington DC, 1996.

20. Kim TH, Ananthaswamy HN, Kripke ML and Ullrich SE: Advantages of using hairless mice versus haired mice to test sunscreen efficacy against photoimmune suppressions. Photochem Photobiol 78: 37-42, 2003.

21. Gibbs NK and Norval M: Photoimmunosuppression: A brief overview. Photodermatol Photoimmunol Photomed 29: 57-64, 2013.

22. Hunger RE, Sieling PA, Ochoa MT, Sugaya M, Burdick AE, Rea TH, Brennan PJ, Belisle JT, Blauvelt A, Porcelli SA and Modlin RL: Langerhans cells utilize CD1a and langerin to efficiently present nonpeptide antigens to T cells. J Clin Invest 113: 701-708, 2004.
23. Slavik JM, Hutchcroft JE and Bierer BE: CD28/CTLA-4 and CD80/CD86 families: Signaling and function. Immunol Res 19: $1-24,1999$.

24. Laihia JK and Jansen CT: Up-regulation of human epidermal Langerhans' cell B7-1 and B7-2 co-stimulatory molecules in vivo by solar-simulating irradiation. Eur J Immunol 27: 984-989, 1997. 25. Fujihara M, Takahashi TA, Azuma M, Ogiso C, Maekawa TL, Yagita H, Okumura K and Sekiguchi S: Decreased inducible expression of CD80 and CD86 in human monocytes after ultraviolet-B irradiation: Its involvement in inactivation of allogenecity. Blood 87: 2386-2393, 1996. 